\title{
Potential of dendrimers as oral drug delivery carriers
}

\author{
P.V. Ravikanth ${ }^{1,2^{*}}$, K.V. Ramanamurthy ${ }^{2}$ \\ ${ }^{1}$ Department of Pharmaceutics, University of Utah, Salt Lake City, UT 84112, USA. \\ ${ }^{2}$ University College of Pharmaceutical Sciences, Andhra University, Visakhapatnam-530 003, India.
}

\begin{tabular}{l}
\hline ARTICLE INFO \\
\hline Article history: \\
Received on: $12 / 03 / 2018$ \\
Accepted on: 09/05/2018 \\
Available online: $30 / 07 / 2018$ \\
\hline Key words: \\
PAMAM Dendrimers, \\
Challenges in oral delivery, \\
Techniques to improve oral \\
bioavailability. \\
\hline
\end{tabular}

\section{INTRODUCTION}

The novel drug delivery strategies aimed at controlling the spatial and temporal distribution of drug molecules in the body by attaching therapeutic compounds to "carriers" by physical and chemical mechanisms in order to improve therapeutic effect and reduce toxicity. The drug molecule with carrier alters pharmacokinetic profile of the drugs hence absorption, distribution, and metabolism, excretion (ADME) of a drug with and without a carrier are different.

Nanomaterials used as drug delivery systems also termed nanocarriers, can contain several moieties to enhance the delivery of bioactive and diagnostic agents. These include targeting ligands for active carrier delivery to the site of action and imaging agents for detection of the nanocarriers in the body, enhanced cell permeation as well as biodegradable linkers for drug release (Larson et al., 2012).

Since most of the emerging drugs are hydrophobic in nature, water-soluble polymers have been tried as carriers to conjugate multiple drug substances, ligands, and diagnostic

\footnotetext{
${ }^{*}$ Corresponding Author

P. Venkata Ravikanth, Department of Pharmaceutics, University of Utah, Salt Lake City,UT 84112,USA.E-mail: u6002241@ utah.edu or venkataravikanth.9@gmail.com
}

agents. These alterations change the fate of the drug in the body and help to specifically target the drugs to the site of action.

Some examples of water-soluble polymers conjugated with drugs tested in the clinic include dendrimers, poly (ethylene) glycol (PEG), $N$-(2-hydroxypropyl) methacrylamide (HPMA) copolymers, and poly (glutamic acid) (PGA) (Tong et al., 2009). Several polymeric delivery systems are now either in clinical trials or on the market for the treatment of cancer and other diseases. Recently PCBP2 siRNA, a potential antifibrotic agent which is Neutravidin based siRNA nanocomplex was prepared in order to target insulin-like growth factor 2 receptor (IGF2R) that is overexpressed in activated hepatic satellite cells (HSCs) (Zhao et al., 2018).

There are different techniques reported to improve permeability of drugs through gastrointestinal tract (GIT) membrane among those techniques permeability enhancement by inhibiting Pgp was studied extensively. P-glycoprotein (Pgp) also known as cluster of differentiation 243 (CD243) or multidrug resistance protein 1 (MDR1) or ATP-binding cassette sub-family $\mathrm{B}$ member 1 (ABCB1) is present in the cell membranes of most of the cells. This protein expression is significantly high at the mucosal side of intestine for absorption of nutrients and excretion of exogenous substances.

This pump recognizes molecules with hydrophobic 
aromatic and/or tertiary maino groups. Several methods are proposed to inhibit the activity of Pgp. For example, verapamil, cyclosporine A, LY335979 are found to inhibit Pgp function and increase the intestinal permeability of drugs including designing competitive inhibitors for Pgp, designing of drugs to avoid recognition by Pgp and complete inactivation of the Pgp function (Zacherl et al., 1994). Surfactants such as polysorbate 80, cremophors were found to inhibit Pgp function and enhance drug transport. This article will provide the pertinent background of dendrimers and their potential for oral delivery in formulation perspective.

\section{DENDRIMERS IN DRUG DELIVERY}

Many of man's innovations seem to draw inspiration from nature. As the Greek word "Dendron" suggests, dendrimers are one such inspiration from a branched tree-like structure. Dendrimers are repeatedly branched macromolecules characterized by structural definition and medicine. Buhleier synthesized the very first dendron-like structure in pursuit of chemically challenging macromolecules that had large molecular cavities. Tomalia and coworkers synthesized the first dendrimers, as we know them today (Abbasi et al., 2014).

Dendritic architecture is the fourth class of polymer architectures created after linear, cross-linked and branched types. It is recognized that delivery of potent drugs to specific sites is essential to avoid the deleterious side effects of these nonspecific agents. Dendrimers form a significant section of polymers employed in drug delivery and nanomedicine. These unique polymers possess many chemical branches that are stretched outward facilitating attachment of the drug payloads. Numerous surface functional groups on the dendrimers also facilitate attachment of targeting moieties and other biorecognizable motifs that augment therapeutic efficacy. The void space between dendritic branches has also been utilized to entrap drugs. The unique dendritic architecture allows reactive agents to be presented on the surface, making them accessible to biological interactions. Chemical synthesis of branched dendrimers confers a high degree of monodispersity ensuring near uniform and welldefined structures (Svenson, 2009).

Other desirable features of these highly symmetric polymers include robustness, high solubility in aqueous media (depending on chemical composition) and biocompatibility. Biocompatibility represents nonimmunogenic and nontoxic properties for medical use. Depending on the type of chemical constituents, dendrimers may vary from non-immunogenic to slightly immunogenic. Toxicity of dendrimers is generation and surface charge dependent. Cationic dendrimers are prone to be more toxic than anionic dendrimers presumably causing nanoscale holes in the plasma membrane of cells (Wiwattanapatapee, 2000).

Different combinations of the core, monomer units, and surface functionality results in the emergence of more than hundreds of dendrimers. Table 1 lists major classes of dendrimers based on their chemical structure and also their use in drug delivery applications.

Table 1: Types of Dendrimers in drug delivery.

\begin{tabular}{|c|c|c|c|}
\hline Chemical structure of dendrimers & Drugs associated & Drug delivery application & Reference \\
\hline \multirow{3}{*}{ Poly(amido amide) (PAMAM) } & Indomethacin & Anti-arthritic & Chauhan et al., 2004 \\
\hline & Sulfamethoxazole & Antibacterial & Ma et al., 2007 \\
\hline & Camptothecin & Treatment against colorectal carcinoma & Sadekar et al., 2013 \\
\hline Poly(propyleneimine) (PPI) & Doxorubicin & Treatment against lung epithelial cancers & Golshan et al., 2007 \\
\hline Poly (L-lysine) & Camptothecin & Treatment against colon cancers Anti-angiogenic & Fox et al., 2009 \\
\hline Triazine & Paclitaxel & Anticancer drug delivery system & Lee et al., 2013 \\
\hline
\end{tabular}

Though there is a variety of dendrimers PPI, PEI and PAMAM dendrimers are thoroughly investigated and found to have widespread applications. PPI stands for Poly (Propylene Imine) with primary amines as end groups and tertiary trispropylene amines in the core. PEI dendrimers which are a subclass of PPI dendrimers contain diamino ethane core and Poly (Ethylene Imine) branches.

PAMAM-dendrimers are the most common class of dendrimers consists of alkyl diamine core and tertiary amine branches. The first dendrimer made and scaled-up were poly (amidoamine) (PAMAM) dendrimers under the name of "starburst". These dendrimers were discussed in detail in view of their widespread applications in biology (Haag et al., 2006). The most frequently used dendrimers are given in Figure 1.

Polyester, polypeptide, triazine and polyglycerol dendrimers are organic dendrimers that have been attached to therapeutics to increase the efficacy of treatment. Many other useful chemical compositions such as the poly (ester acrylate/ amide) (PEA) and poly(etherhydroxylamine) (PEHAM) are commercially available and used in biomedical applications. Hybrid structures of dendrimers with other entities have been synthesized for their collective properties. Most common is PEGylation of dendrimers to increase their circulation in the blood and to avoid immune clearance. Lipids have also been attached to the surface of PAMAM dendrimers to reduce toxicity and increase cellular uptake.

Incorporation of inorganic molecules into dendrimer structures has shown distinct advantages. For example, the recent carbosilane dendrimers with surface lactose entities seem to possess inherent antiviral properties. In another case, fluorine molecules were incorporated into the core of dendrimers that offer potential in various fields such as materials, catalysis, and biology.

\section{PAMAM dendrimers}

Poly (amidoamine) (PAMAM) dendrimers are class of water-soluble polymers that demonstrate potential in oral drug administration. PAMAM dendrimers also called as Starburst ${ }^{\mathrm{TM}}$ dendrimers and they are characterized by a unique tree-like branching architecture that starts from an initiator core around which the branches originates. Each series of this branching mechanism is termed a generation $(\mathrm{G})$ and an increase in generation results in an incremental increase in dendrimer size, mass, geometry, and 
surface functionality. PAMAM dendrimers are promising drug delivery systems due to the high degree of "branches" that can be terminated with a cationic amine $\left(-\mathrm{NH}_{2}\right)$, neutral hydroxyl ($\mathrm{OH})$, or anionic carboxylic acid $(-\mathrm{COOH})$ surface groups, which allow the conjugation of biological agents in a compact system. A variety of molecules, such as drugs and other therapeutic agents,

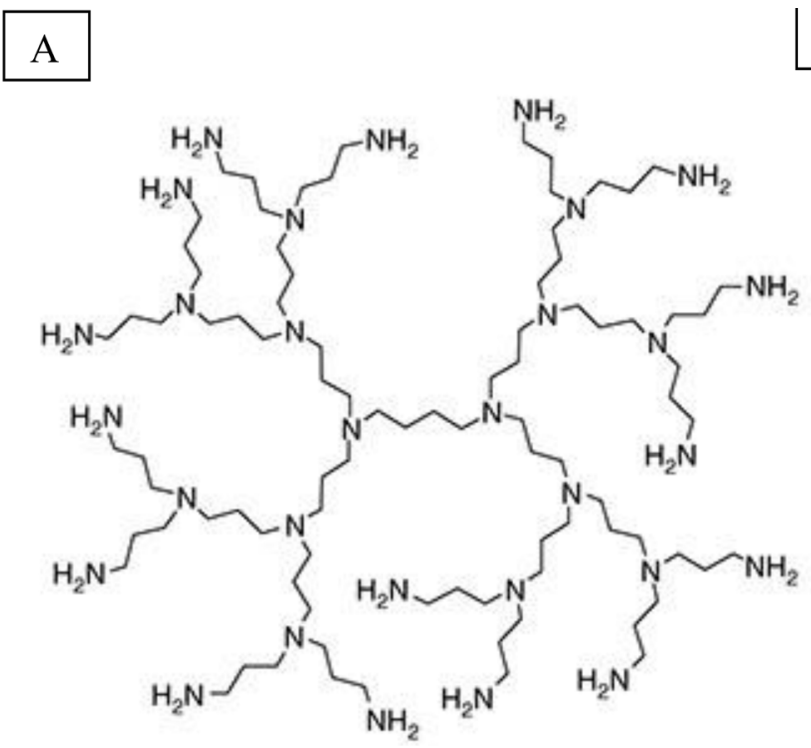

can be loaded both in the interior void space and on the surface functional groups to control the release rate of these agents in the body. PAMAM dendrimers are commercially available, usually as methanol solutions up to generation 11 (Prabal et al., 2004). These macromolecules have permeability issue as discussed in detail below.

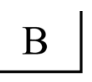

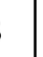

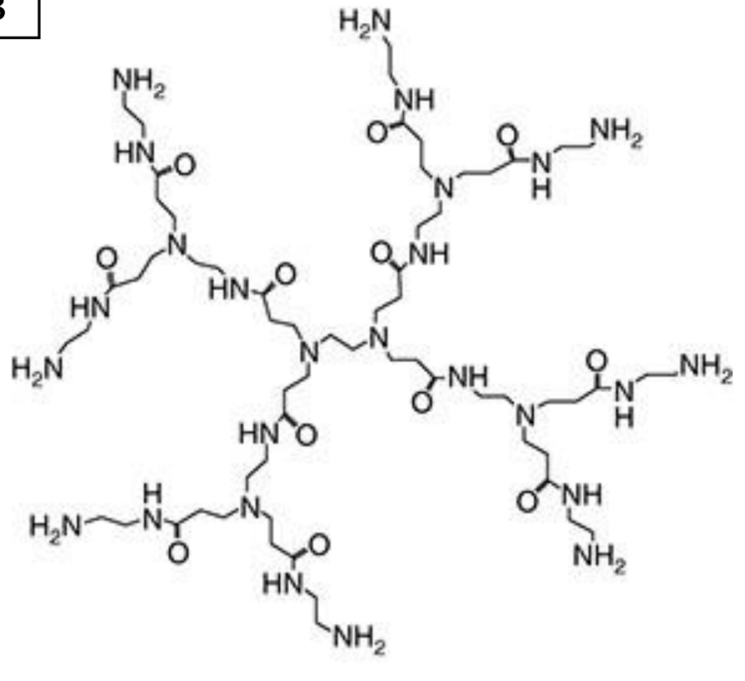

Fig. 1: Frequently used dendrimers for biological applications (A) PPI (B) PAMAM. (Reprinted with permission from Knecht et al., 2004).

\section{CHALLENGES IN ORAL DELIVERY OF DENDRIMERS}

A minimum level of lipophilicity is required for drugs to partition into epithelial cell membranes and get absorbed transcellularly through passive diffusion. Poor bioavailability of the drug is attributed primarily to large molecular size and solubility characteristics of the drug. Bioavailability decreases sharply beyond the molecular mass of 500-700 Daltons (Donovan et al., 1990) but below this molecular mass bioavailability is independent of drugs solubility characteristics.

Table 2 is the list of various generations of PAMAM dendrimers with their corresponding sizes. Due to the size of the dendrimers, there should be some permeation enhancers included in the formulation.

Table 2: Size of PAMAM dendrimers with $\mathrm{NH}_{2}$ functional groups.

\begin{tabular}{cccc}
\hline Generation & Surface groups & Molecular formula & Diameter (nm) \\
\hline 0 & 4 & $\mathrm{C}_{24} \mathrm{H}_{52} \mathrm{~N}_{12} \mathrm{O}_{4} \mathrm{~S}_{2}$ & 1.4 \\
1 & 8 & $\mathrm{C}_{64} \mathrm{H}_{132} \mathrm{~N}_{26} \mathrm{O}_{12} \mathrm{~S}_{2}$ & 1.9 \\
2 & 16 & $\mathrm{C}_{144} \mathrm{H}_{292} \mathrm{~N}_{58} \mathrm{O}_{28} \mathrm{~S}_{2}$ & 2.6 \\
3 & 32 & $\mathrm{C}_{304} \mathrm{H}_{612} \mathrm{~N}_{122} \mathrm{O}_{60} \mathrm{~S}_{2}$ & 3.6 \\
4 & 64 & $\mathrm{C}_{624} \mathrm{H}_{1252} \mathrm{~N}_{250} \mathrm{O}_{124} \mathrm{~S}_{2}$ & 4.4 \\
5 & 128 & $\mathrm{C}_{1264} \mathrm{H}_{2532} \mathrm{~N}_{506} \mathrm{O}_{252} \mathrm{~S}_{2}$ & 5.7 \\
6 & 256 & $\mathrm{C}_{2544} \mathrm{H}_{5092} \mathrm{~N}_{1018} \mathrm{O}_{508} \mathrm{~S}_{2}$ & 7.2 \\
\hline
\end{tabular}

Oral administration of protein therapeutics has always been a challenge, due to enzymatic degradation in GIT. Attempts have been made to decrease this protein degradation by conjugating these therapeutics to dendrimers and co-administering them with enzyme inhibitors (Muheem et al., 2016). Bioavailability of these conjugates further improved using only permeability enhancers which is essential not only to increase bioavailability but also decrease residence time in the GIT where it gets degraded enzymatically.

It was found in earlier studies that the mechanism of cellular uptake and results indicate that clathrin-dependent endocytic pathways play a major role in the internalization of PAMAM dendrimers. Surface charge, concentration and generation influence the uptake of dendrimers (Kitchen et al., 2006). When ligands or other targeting molecules form a part of the system, they are taken up by receptor-mediated endocytosis. Here; the targeting moieties bind to receptors on the cell surface and trigger receptor-mediated endocytosis. Drugs and other molecules attached to the dendrimer might influence the rate of uptake depending on the degree of hydrophobicity and charge.

\section{TECHNIQUES TO IMPROVE GIT PERMEABILITY OF DENDRIMERS}

The approach for modifying epithelial permeability fall into two main categories: those that increase cell-membrane permeability or cause "membrane perturbation" in order to enhance transcellular transport, and those that target the tight junctions between cells in order to increase paracellular absorption as shown in the Figure 2. Lipids are known to play an important role in TJ modulation are also involved in lipid exchanges that occur between apical regions of adjacent epithelial cells. Cholesterol forms an important component of these lipid structures, and depleting these molecules from the TJs leads to junctional permeability.

These approaches are limited as the opening of tight junction not only permeabilize drug but also potentially toxic 
substances present in the GIT. Hence the optimum amount of surfactant should be taken which can be found using cell cytotoxicity and/or using chamber studies.

\section{Cell membrane permeabilization}

Medium-to-long-chain fatty acids including $\mathrm{C}_{8}, \mathrm{C}_{10}$, sodium8-([2-hydroxybenzoyl] amino) octanoate, acetyl carnitines and bile salts with citric acids are now in clinical evaluation for their proposed enhancement of oral drugs such as insulin, GLP-1, calcitonin, etc. Pharmaceutical companies such as Novo Nordisk (Denmark), MerrionPharma (Ireland), Emisphere technologies (USA) and Biocon (India) are currently evaluating these technologies. These agents act as nonionic surfactants in increasing the intestinal membrane permeability. The successful commercialization of these technologies is yet to be materialized.

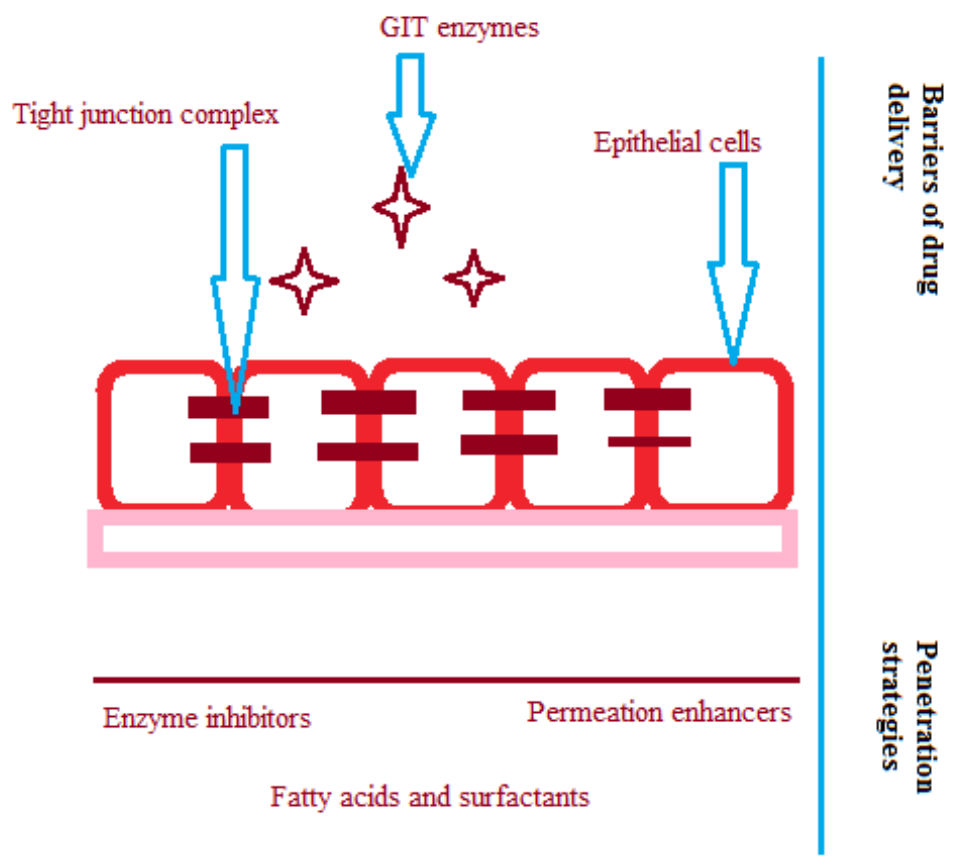

Fig. 2: Schematic representation of pathways of permeability of therapeutics.

\section{Tight-junction modifications}

Vitamin E-TPGS and Capmul MCM are two excipients used to increase the permeability of drugs by opening the tight junction in the GIT membrane. These two formulations have got generally regarded as safe (GRAS) status hence abundantly investigated in the researches.

Vitamin E-TPGS is a novel nonionic surfactant synthesized by the esterification of Vitamin E succinate with polyethylene glycol 1000. Being a surfactant with low critical micellar concentration (CMC) of $0.02 \mathrm{wt} \%$ it can form stable micelles in the aqueous vehicles. It has been tried for emulsifying, dispersing and solubilizing various poorly water-soluble and permeable drugs. The permeability of therapeutics found to be improved by modulating the tight junction of the epithelium of intestine and inhibiting P-glycoprotein (P-gp).

Capmul ${ }^{\circledR}$ MCM is chemically mono-diglyceride of medium chain fatty acids (mainly caprylic and capric). It was widely investigated for its ability to work as permeability enhancer. It is an excellent solvent for many hydrophobic substances and works as emulsifier in water-oil (w/o) emulsions.

Tight junction modulators such as Zonulaoccludens toxin and its mimics, Pz-peptides, nitric oxide donors, Ethylenediaminetetraacetic acid (EDTA) and chitosans clostridium perfingins enterotoxin act on components of tight junction proteins and directly or intracellularly influence reversible opening of tight junctions between epithelial cells (Maher et al., 2016). For reasons such as stability, potency, toxicity or immune clearance, these agents have not achieved convincing preclinical and significant bioavailability of therapeutic peptides. Nevertheless, academic studies on various models show impressive mechanistic and bioavailability data including amphiphilic polymeric peptide complexes, peptides in w/o and w/o/w emulsions and in aqueous core nanocapsules.

\section{Receptor-mediated endocytosis}

Receptor-based targeting techniques to improve transcellular uptake have also been tried by various groups. The rationale behind this is that ligands for well-expressed receptors on small intestinal cell surface will increase their uptake substantially. Target receptors include hPEPt1, FcRn, TfnR, hSMVT, cubulin, ICAM-1 or CD54, LRP1 or CD91 and ganglioside GM1 (Brayden et al., 2012). The drawback with these approaches is the consistent production of formulations with these targeting motifs and the stability in the harsh GI environment due to high protease load, a variation of receptor expression between patients, etc. Vitamin $\mathrm{B}_{12}$ is a receptormediated strategy that is currently being pursued. 


\section{Increasing lipophilicity}

Covalent attachment of lipophilic molecules as sodium N-[8-(2-hydroxybenzoyl) amino] caprylate (SNAC) or salcaprozate sodium, also called sodium $\mathrm{N}$-[8-(2-hydroxybenzoyl) amino] octanoatethat was patented by Eligen technology (salcaprozatesodium, etc.) (Emisphere Technologies, NJ, USA) has shed a light in the improvement of permeability. In addition to these techniques submicron-size particles, nanoparticles and liposomes have been evaluated earlier for oral absorption through transcytosis by both enterocytes and M-cell.

\section{Future outlook}

Oral delivery remains the most patient compliant route for therapeutics. Polymer-based drug delivery has overcome many challenges like solubility and permeability issues associated with the drug and route. Dendrimers are one of such polymers helped to overcome such challenges. But dendrimers have the major drawback of toxicity which is associated with its charge. Surface modification of these dendrimers using novel chemistries should overcome this issue.

\section{CONCLUSION}

Dendrimers have the advantage of transporting a large number of drug molecules both orally and intravenously. They have limited applications in oral administration since they are unable to cross the tight junctions of the intestinal epithelium. But, applying various permeability enhancement techniques discussed earlier will improve the permeability when administered orally. Cell membrane permeabilization, tight junction modulation, and Eligen technology are efficient techniques for enhancing the permeability.

\section{REFERENCES}

Larson N, Ghandehari H. Polymeric conjugates for drug delivery. Chem Mater, 2012; 24(5):840-53.

Tong R, Christian DA, Tang L, Cabral H, Baker JR, Kataoka K, Discher DE, Cheng J. Nanopolymeric therapeutics. Mrs Bulletin, 2009; 34(6):422-31.

Zhao Z, Li Y, Jain A, Chen Z, Liu H, Jin W, Cheng K. Development of a peptide-modified siRNAnanocomplex for hepatic stellate cells. Nanomedicine, 2018; 14(1):51-61.

Zacherl J, Hamilton G, Thalhammer T, Riegler M, Cosentini EP, Ellinger A, Bischof G, Schweitzer M, Teleky B, Koperna T, Wenzl E. Inhibition of P-glycoprotein-mediated vinblastine transport across HCT-8 intestinal carcinoma monolayers by verapamil, cyclosporine A and SDZPSC 833 in dependence on extracellular pH. Cancer Chemother Pharmacol, 1994; 34(2):125-32.

Abbasi E, Aval SF, Akbarzadeh A, Milani M, NasrabadiHT, Joo SW, Hanifehpour Y, Nejati-Koshki K, Pashaei-Asl R. Dendrimers: synthesis, applications, and properties. Nanoscale Res Lett, 2014; 9(1):247.

Svenson S. Dendrimers as versatile platform in drug delivery applications. Eur J Pharm Biopharm, 2009; 71(3):445-62.

Wiwattanapatapee R, Carreño-Gómez B, Malik N, Duncan R. Anionic PAMAM dendrimers rapidly cross adult rat intestine in vitro: a potential oral delivery system? Pharm Res, 2000; 17(8):991-8.

Chauhan AS, Jain NK, Diwan PV, Khopade AJ. Solubility enhancement of indomethacin with poly (amidoamine) dendrimers and targeting to inflammatory regions of arthritic rats. J drug target, 2004; $12(9-$ 10):575-83.

Ma M, Cheng Y, Xu Z, Xu P, Qu H, Fang Y, Xu T, Wen L. Evaluation of polyamidoamine (PAMAM) dendrimers as drug carriers of anti-bacterial drugs using sulfamethoxazole (SMZ) as a model drug. Eur J Med Chem, 2007; 42(1):93-8.

Sadekar S, Thiagarajan G, Bartlett K, Hubbard D, Ray A, McGill LD, Ghandehari H. Poly (amido amine) dendrimers as absorption enhancers for oral delivery of camptothecin. Int J Pharm, 2013; 456(1):175-85.

Golshan M, Salami-Kalajahi M, Mirshekarpour M, RoghaniMamaqani H, Mohammadi M. Synthesis and characterization of poly (propylene imine)-dendrimer-grafted gold nanoparticles as nanocarriers of doxorubicin. Colloids Surf B Biointerfaces, 2017; 155:257-65.

Fox ME, Guillaudeu S, Fréchet JM, Jerger K, Macaraeg N, Szoka FC. Synthesis and in vivo antitumor efficacy of PEGylated poly (1-lysine) dendrimercamptothecin conjugates. Mol Pharm, 2009; 6(5):1562-72.

Lee C, Lo ST, Lim J, da Costa VC, Ramezani S, Öz OK, Pavan GM, Annunziata O, Sun X, Simanek EE. Design, synthesis and biological assessment of a triazine dendrimer with approximately 16 paclitaxel groups and 8 PEG groups. Mol Pharm, 2013; 10(12):4452-61.

Haag R, Kratz F. Polymer therapeutics: concepts and applications. Angew Chem Int Ed Engl, 2006; 45(8):1198-215.

Knecht MR, Wright DW. Amine-terminated dendrimers as biomimetic templates for silica nanosphere formation. Langmuir, 2004; 20(11):4728-32.

Maiti PK, Çağın T, Wang G, Goddard WA. Structure of PAMAM dendrimers: Generations 1 through 11. Macromolecules, 2004; 37(16):6236-54.

Donovan MD, Flynn GL, Amidon GL. Absorption of polyethylene glycols 600 through 2000: the molecular weight dependence of gastrointestinal and nasal absorption. Pharm Res, 1990; 7(8):863-8.

Muheem A, Shakeel F, Jahangir MA, Anwar M, Mallick N, Jain GK, Warsi MH, Ahmad FJ. A review on the strategies for oral delivery of proteins and peptides and their clinical perspectives. Saudi Pharm J, 2016; 24(4):413-28.

Kitchens KM, Kolhatkar RB, Swaan PW, Eddington ND, Ghandehari H. Transport of poly (amidoamine) dendrimers across Caco-2 cell monolayers: influence of size, charge and fluorescent labeling Pharm Res, 2006; 23(12):2818-26.

Maher S, Mrsny RJ, Brayden DJ. Intestinal permeation enhancers for oral peptide delivery. Adv Drug Deliv Rev, 2016; 106:277-319.

Brayden DJ, Mrsny RJ. Oral peptide delivery: prioritizing the leading technologies. Ther Deliv, 2011; 2(12):1567-73.

How to cite this article:

Ravikanth PV, Ramanamurthy KV. Potential of dendrimers as oral drug delivery carriers. J App Pharm Sci, 2018; 8(07): 149153. 